Article

\title{
Synthesis and Characterization of Aminophosphonate Containing Chitosan Polymer Derivatives: Investigations of Cytotoxic Activity and in Silico Study of SARS-CoV-19
}

\author{
Ponnusamy Packialakshmi ${ }^{1}$, Perumal Gobinath ${ }^{1}$, Daoud Ali ${ }^{2} \oplus$, Saud Alarifi ${ }^{2} \oplus$, Norah Salem Alsaiari ${ }^{3}$, \\ Akbar Idhayadhulla ${ }^{1}\left(\mathbb{D}\right.$ and Radhakrishnan Surendrakumar ${ }^{1, *(1)}$ \\ 1 PG \& Research, Department of Chemistry, Nehru Memorial College, Affiliated Bharathidasan University, \\ Puthanamapatti, Tamilnadu 621007, India; packiyaponnusamy96@gmail.com (P.P.); \\ gopi10207005@gmail.com (P.G.); idhayadhulla@nmc.ac.in (A.I.) \\ 2 Department of Zoology, College of Sciences, King Saud University (KSU), P.O. Box 2455, \\ Riyadh 11451, Saudi Arabia; aalidaoud@ksu.edu.sa (D.A.); salarifi@ksu.edu.sa (S.A.) \\ 3 Department of Chemistry, College of Science, Princess Nourah Bint Abdulrahman University, \\ Riyadh 11451, Saudi Arabia; nsalsaiari@pnu.edu.sa \\ * Correspondence: surendrakumar@nmc.ac.in; Tel.: +91-99-4454-9026
}

check for updates

Citation: Packialakshmi, P.; Gobinath, P.; Ali, D.; Alarifi, S.; Alsaiari, N.S.; Idhayadhulla, A.; Surendrakumar, R. Synthesis and Characterization of a

Minophosphonate Containing Chitosan Polymer Derivatives: Investigations of Cytotoxic Activity and in Silico Study of SARS-CoV-19. Polymers 2021, 13, 1046. https:// doi.org/10.3390/polym13071046

Academic Editor: Muhammad S. Zafar

Received: 24 February 2021

Accepted: 15 March 2021

Published: 26 March 2021

Publisher's Note: MDPI stays neutral with regard to jurisdictional claims in published maps and institutional affiliations.

Copyright: (C) 2021 by the authors Licensee MDPI, Basel, Switzerland. This article is an open access article distributed under the terms and conditions of the Creative Commons Attribution (CC BY) license (https:// creativecommons.org/licenses/by/ $4.0 /)$
Abstract: Chitosan is broadly used as a biological material since of its excellent biological activities. This work describes investigations of chitosan interaction with SARS-CoV-2, which is occupied by human respiratory epithelial cells through communication with the human angiotension-converting enzyme II (ACE2). The $\beta$-chitosan derivatives are synthesized and characterized by FT-IR, nuclear magnetic resonance $\left({ }^{1} \mathrm{H}\right.$ and $\left.{ }^{13} \mathrm{C} \mathrm{NMR}\right)$, mass spectrometry, $\mathrm{X}$-ray diffraction, TGA, DSC, and elemental analysis. The $\beta$-chitosan derivatives were screened for cytotoxic activity against the HepG2 and MCF-7 (breast) cancer cell lines. Compound $\mathbf{1 h}\left(\mathrm{GI}_{50} 0.02 \mu \mathrm{M}\right)$ is moderately active against the HepG2 cancer cell line, and Compound 1c is highly active $\left(\mathrm{GI}_{50} 0.01 \mu \mathrm{M}\right)$ against the MCF-7 cancer cell line. In addition, chitosan derivatives (1a-1j) docking against the SARS coronavirus are found by in-silico docking analysis. The findings show that compound 1c exhibits notable inhibition ability compared with other compounds, with a binding energy value of $-7.9 \mathrm{kcal} / \mathrm{mol}$. Based on the molecular docking results, the chitosan analog is proposed to be an alternative antiviral agent for SARS-CoV2.

Keywords: chitosan; Schiff base; spectral analysis; X-ray diffraction; TGA; DSC and cytotoxic activity; In silico analysis

\section{Introduction}

The reported SARS that turned into COVID-19 was originally a coronavirus, SARSCoV-2. The first cases of SARS-CoV were reported as occurring in November 2002 in China (although not recognized at that time), and the WHO became aware of these in early 2003 [1]. Severe Acute Respiratory Syndrome (SARS) is a novel viral typical pneumonia. This communicable illness was reported on 31 December 2019. The World Health Organization (WHO) was officially informed about cases of pneumonia in Wuhan City, home to 11 million people and the cultural and financial hub of Central China, and afterward quickly extended to 25 other countries [2]. The infection spreads mainly from one person to another through small droplets from the nose or mouth. On 11 August 2020, Russia became the first country in the world to approve a vaccine (Sputnik V) against (SARS-CoV2). The vaccine, which is based on two adenovirus vectors, wasdeveloped by the Gamaleya National Center of Epidemiology and Microbiology (Moscow, Russia) [3], even though steroids and ribavirin have been used in hospitals of Hong Kong [4,5] and HIV protease inhibitor nelfinavir can reduce replication of SARS-CoV based on an initialin vitroassessment [6]. In view of the fact that the SARS CoV 3C-like protease playsa very significant role in the viral 
life cycle, there has been a key goal planned for the structure-based drug proposal against SARS [7]. The drug-resistant and insufficient antiviral drugs have increased demand for the development of more potent and less toxic antiviral agents. Chitosan-based antiviral research was published as shown in Figure 1 [8]. The chemical alteration of chitosan (CS) has recently been paid attention byresearchers due to its wide variety of applications.

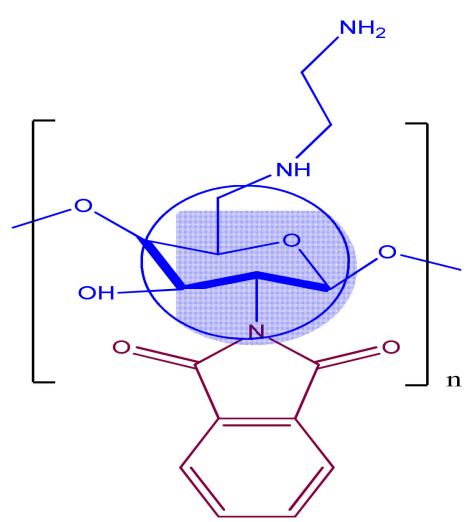

Figure 1. Antiviral activity (Newcastle virus) of chitosan derivatives.

The chemical compositions of chitosan components powerfully influence its biological activity. These factors certainly must be taken into account in an analysis of its antiviral activity [9]. Chitin has a chemical structure of a characteristic supplementary biopolymer found in the exoskeletons of roaches and crustaceans [10], but chitosan is frequently obtained by the deacetylation of chitin [11]. $\mathrm{CS}$ has a few receptive $\mathrm{NH}_{2}$ groups that offer additional chemical alteration for the expansion of derivatives that are costeffective [12]. Deacetylated chitosan is an extremely useful and readily available bioactive polymer $[13,14]$. Chitosan displays several beneficial biological properties, such as antitumor and antimicrobial [15]. The antiviral activity of chitosan is determined by its structure and, by the polymerization degree of the molecule [16]. Thus, the high polymeric preparations of polysaccharides possess antiviral activity. On the other hand, it has been shown that the high polymeric chitosan's significantly increased its antiviral activity $[17,18]$. It consists of unbranched chain of $\beta$-glucose as repeating units [19]. As a result, data on the dependence of chitosan derivative, mainly the polymerization degree, are in significant disagreement, which can be determined by the variety of chitosan sources [20]. Chitosan is depolymerized by chemical hydrolysis, which is performed for obtainingsamples of the polysaccharide. The initial high molecular (HM) samples of chitosan and the methods of its purification also can increase the biological activity of its chitosan derivatives [21]. The polymeric structure of chitosan derivative is given in Figure 2 [22].<smiles>COC(O)C(O)C(O)C(OC)N(CC1CC2CCC1C2)c1coc2ccccc2c1=O</smiles>

Figure 2. Polymeric structure of chitosan derivatives. 
Phosphorylated chitin (P-chitin) and chitosan (P-chitosan) were prepared by the reaction of chitin or chitosan with orthophosphoric acid and urea in DMF [23] with phosphorous pentoxide in methane sulphonic acid [24]. The amino phosphonates are among the most common organophosphorus derivatives and have been used inantiviral, antibacterial, antifungal, and anticancer activities [25-30]. Many of the Schiff base chitosan derivatives exhibit pharmacological properties likeantimicrobial and anticancer shown in Figure 3 [31,32].

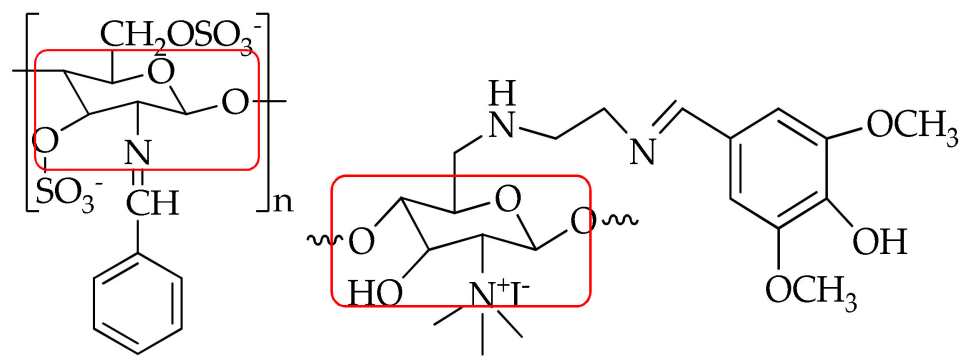

Figure 3. Anticancer activity of chitosan derivatives.

In the current work, new aminophosphonates containing chitosan moieties are synthesized. Particularly, we graft fluorobenzaldehyde, fluoroaniline, and triphenylphosphite in the presence of lithium perchlorate as a catalyst. To the best of our knowledge, no work on a-aminophosphonate and fluoro aniline with $\beta$-chitosan has been previously reported in the literature.

\section{Experimental}

\subsection{Materials and Methods (Chemistry)}

Melting points were recorded in open capillary tubes. The FT-IR spectra wererecorded the $\mathrm{KBr}$ disk technique on a Shimadzu 8201pc $\left(4000-1000 \mathrm{~cm}^{-1}\right)$. The ${ }^{1} \mathrm{H}-\mathrm{NMR}$ and ${ }^{13} \mathrm{C}-$ NMR spectra were recorded on a Bruker DRX-300 MHz. The compound was dissolved in DMSO- $\mathrm{d}_{6}$ to obtain NMR spectra. Mass spectra were recorded by Perkin Elmer GCMS model Clarus SQ8 (EI). The elemental analysis (C, H, and N) was performed using an Elemental analyser model (Varian EL III). The purity of the compounds was checked by TLC with silica gel plates. The starting materials $\beta$-chitosan, substituted aldehydes, and fluoro aniline were attained from Sigma Aldrich, India.

\subsubsection{Preparation of Chitosan Analogue (1a-1j)}

A mixture containing Compound $1(0.001 \mathrm{~mol}, 0.547 \mathrm{~g})$ and 4-fluoroailine $(0.01 \mathrm{~mol}$, $0.947 \mathrm{~mL}$ ) along with $15 \mathrm{~mL}$ ethanol and $\mathrm{NaOH}$ was added withcontinuous stirring at high temperature, and also underwent dehydration to obtain Compound 1a as a white solid. The obtained product was treated with ice-cold water. The progress of the reaction was monitored by TLC. Hexane and ethylacetate (4:6) were used as eluting solvents in TLC. Thin-layer chromatography (TLC) was done by CCM Gel de silica gel 60 F254 sheet $(0.1 \mathrm{~mm})$ with hexane/ethylacetate (4:6 by volume) as emerging eluents. The compounds were detected passing an E-Series UV hand lamp (254/365 nm wave length). The compounds are purified by column chromatography (gel filtration method, wet condition), and silica gel (60-120 mesh) was used as a sorbent. Hexane/ethyl acetate was used an eluting solvents. The obtained solid was further purified by recrystallization from ethanol. The above procedure was followed for the synthesis of Compounds $\mathbf{1 b} \mathbf{1} \mathbf{1 j}$.

Synthesis of diphenylN-(4-fluorophenyl)-P-((1)-(4-fluorophenyl) (((2,3,6)-4-hydroxyl-6(hydroxylmethyl)-2,5-dimethoxytetrahydro-2H-pyran-3-yl)amino)methyl)phosphonimidate (1a) White solid; yield 60\%; mp $108{ }^{\circ} \mathrm{C}$; IR ( $\left.\mathrm{kBr}\right)\left(\mathrm{cm}^{-1}\right)$; $3450(\mathrm{OH}$, str), 3280 (NH-str), 3050 (CH-str Ar ring), 2925 (CH, str), 1425 (N-C, str), 1120 (P=N), 890-900 (P-O-C, str). ${ }^{1} \mathrm{H}$ NMR $\left(\mathrm{CDCl}_{3}\right), \delta(\mathrm{ppm}): 7.95(\mathrm{~s}, 1 \mathrm{H}, \mathrm{NH}), 7.90-7.21(\mathrm{~m}, 4 \mathrm{H}, \mathrm{F}-\mathrm{Ph}), 7.28-7.01\left(\mathrm{~m}, 10 \mathrm{H}, \mathrm{PO}-(\mathrm{Ph})_{3}\right)$, 6.85-6.63 (m, 4H, F-aniline), $5.83(\mathrm{~s}, 2 \mathrm{H}, \mathrm{CS}-\mathrm{OH}), 5.56(\mathrm{~d}, 1 \mathrm{H}, \mathrm{CH}, J=14-17 \mathrm{~Hz}), 4.87(\mathrm{~m}$, 
$5 \mathrm{H}, \mathrm{CS}-\mathrm{H}), 3.50(\mathrm{~d}, 2 \mathrm{H}, \mathrm{CS}, J=10 \mathrm{~Hz}), 3.52(\mathrm{~s}, 6 \mathrm{H}, \mathrm{CS}) \cdot{ }^{13} \mathrm{C} \mathrm{NMR}\left(\mathrm{CDCl}_{3}\right) \delta(\mathrm{ppm}): 161.4$, 116.8, 115.9 (6C, F-Ph), 157.7, 155.3, 142.5, 115.3 (6C, F-Ph-NH $\left.{ }_{2}\right), 152.7,124.9,121.5,121.3$ (12C, PO-(Ph) 3 ), 102.8, 85.6, 76.3, 73.1, 67.6, 60.5, 59.5, 55.7 (8C, CS), 75.6 (1C, CH). EI-MS (relative intensity \%): $m / z 642.22\left(\mathrm{M}^{+}, 20 \%\right)$. Elemental analysis: Anal. $\mathrm{C}_{33} \mathrm{H}_{35} \mathrm{~F}_{2} \mathrm{~N}_{2} \mathrm{O}_{7} \mathrm{P}: \mathrm{C}$, 61.87; H, 5.51; N, 4.35; Found, C, 61.88; H, 5.50; N, 4.37.

Diphnyl-P-((1)-(4-chlorophenyl)(((2,3,6)-4-hydroxy-6-(hydroxymethyl)-2,5dimethoxytetrahdro-2H-pyran-3-yl)amino)methyl)-N-(4-fluorophenyl)phosphonimidate (1b) Light yellow solid; yield 70\%; mp120 ${ }^{\circ} \mathrm{C}$; IR ( $\left.\mathrm{kBr}\right)\left(\mathrm{cm}^{-1}\right)$; $3420(\mathrm{OH}$, str), 3250 (NH-str), 3030 (CH-str Ar ring), 2935 (CH, str), 1372 (N-C, str), 1120 (P=N), 900 (P-O-C, str). ${ }^{1} \mathrm{H}$ NMR $\left(\mathrm{CDCl}_{3}\right), \delta(\mathrm{ppm}): 9.28(\mathrm{~s}, 1 \mathrm{H}, \mathrm{NH}), 7.95-7.52(\mathrm{~m}, 4 \mathrm{H}, \mathrm{Cl}-\mathrm{Ph}), 7.26-7.00\left(\mathrm{~m}, 10 \mathrm{H}, \mathrm{PO}-(\mathrm{Ph})_{3}\right)$, 6.83-6.60 (m, 4H, F-aniline), $5.85(\mathrm{~s}, 2 \mathrm{H}, \mathrm{CS}-\mathrm{OH}), 5.62(\mathrm{~d}, 1 \mathrm{H}, \mathrm{CH}, J=15 \mathrm{~Hz}), 4.85(\mathrm{~m}, 5 \mathrm{H}$, CS-H), $3.52(\mathrm{~d}, 2 \mathrm{H}, \mathrm{CS}, J=10 \mathrm{~Hz}), 3.50(\mathrm{~s}, 6 \mathrm{H}, \mathrm{CS}) .{ }^{13} \mathrm{C} \mathrm{NMR}\left(\mathrm{CDCl}_{3}\right) \delta(\mathrm{ppm}): 157.5,155.6$, 142.3, 115.7 (6C, F-Ph- $\mathrm{NH}_{2}$ ), 152.4, 124.5, 121.7, 121.6 (12C, PO-(Ph) $)$ ), 135.4, 125.3, 120.4, 116.5 (6C, Cl-Ph), 102.3, 85.5, 76.7, 73.4, 67.2, 60.1, 59.6, 55.5 (8C, CS), 75.3 (1C, CH). EI-MS (relative intensity \%): $m / z 658.18\left(\mathrm{M}^{+}, 21 \%\right)$. Elemental analysis: Anal. $\mathrm{C}_{33} \mathrm{H}_{35} \mathrm{ClFN}_{2} \mathrm{O}_{7} \mathrm{P}$ : C, 60.32; H, 5.32; N, 4.26; Found, C, 60.34; H, 5.33; N, 4.25.

DiphenylN-(4-fluorophenyl)-P-((1)-(((2,3,6)-4-hydroxy-6-(hydroxymethyl)2,5-dimethoxytetrahydro-2H-pyran-3-yl)amino)(4-hydroxyphenyl)methyl)phosphonimidate (1c) Light yellow powder; yield $78 \%$; mp $138{ }^{\circ} \mathrm{C}$; $\mathrm{IR}(\mathrm{kBr})\left(\mathrm{cm}^{-1}\right) ; 3405(\mathrm{OH}, \mathrm{str}), 3252(\mathrm{NH}-$ str), 3033 (CH-str Ar ring), 2937 (CH, str), 1372 (N-C, str), 1120 (P=N), 905 (P-O-C, str). ${ }^{1} \mathrm{H}$ NMR $\left(\mathrm{CDCl}_{3}\right), \delta(\mathrm{ppm}): 10.60$ (s, 1H, Ph-OH), 9.34 (s, $\left.1 \mathrm{H}, \mathrm{NH}\right), 7.75,(\mathrm{~m}, 4 \mathrm{H}, \mathrm{OH}-\mathrm{Ph})$, 7.24-6.98 (m, 10H, PO-(Ph) $\left.)_{3}\right), 6.87(\mathrm{~m}, 4 \mathrm{H}, \mathrm{F}$-aniline), $5.82(\mathrm{~s}, 2 \mathrm{H}, \mathrm{CS}-\mathrm{OH}), 5.58(\mathrm{~d}, 1 \mathrm{H}$, $\mathrm{CH}, J=15 \mathrm{~Hz}), 4.80-3.85(\mathrm{~m}, 5 \mathrm{H}, \mathrm{CS}-\mathrm{H}), 3.55(\mathrm{~d}, 2 \mathrm{H}, \mathrm{CS}, J=10 \mathrm{~Hz}), 3.53(\mathrm{~s}, 6 \mathrm{H}, \mathrm{CS}) .{ }^{13} \mathrm{C}$ NMR $\left(\mathrm{CDCl}_{3}\right) \delta(\mathrm{ppm}): 161.6,129.9,120.3,116.0(6 \mathrm{C}, \mathrm{OH}-\mathrm{Ph}), 157.4,155.7,142.4,115.8(6 \mathrm{C}$, F-Ph- $\left.\mathrm{NH}_{2}\right), 152.6,124.7,121.8,121.4$ (12C, PO-(Ph) 3$), 102.4,85.6,76.8,73.5,67.2,60.3,59.7$, $55.3(8 \mathrm{C}, \mathrm{CS}), 75.1(1 \mathrm{C}, \mathrm{CH})$. EI-MS (relative intensity \%): $m / z 640.23\left(\mathrm{M}^{+}, 19 \%\right)$. Elemental analysis: Anal. $\mathrm{C}_{33} \mathrm{H}_{36} \mathrm{FN}_{2} \mathrm{O}_{8} \mathrm{P}$ : C, 62.06; $\mathrm{H}, 5.68 ; \mathrm{N}, 4.39$; Found, $\mathrm{C}, 62.07 ; \mathrm{H}, 5.69 ; \mathrm{N}, 4.40$.

DiphenylN-(4-fluorophenyl)-P-((1)-(((2,3,6)-4-hydroxy-6-(hydroxymethyl)2,5-dimethoxytetrahydro-2H-pyran-3-yl)amino)(4methoxyphenyl)methyl)phosphonimidate (1d) White solid; yield 65\%; mp $146{ }^{\circ} \mathrm{C}$; IR ( $\left.\mathrm{kBr}\right)\left(\mathrm{cm}^{-1}\right)$; $3435(\mathrm{OH}$, str), 3225 (NH-str), 3038 (CH-str Ar ring), 2931 (CH, str), 1378 (N-C, str), 1173 ( $\mathrm{P}=\mathrm{N}), 903$ (P-O-C, str). ${ }^{1} \mathrm{H}$ NMR $\left(\mathrm{CDCl}_{3}\right), \delta(\mathrm{ppm}): 9.25(\mathrm{~s}, 1 \mathrm{H}, \mathrm{NH}), 7.85-6.99\left(\mathrm{~m}, 4 \mathrm{H}, \mathrm{OCH}_{3}-\mathrm{Ph}\right), 7.07-6.93(\mathrm{~m}, 10 \mathrm{H}, \mathrm{PO}-$ $\left.(\mathrm{Ph})_{3}\right), 6.89-6.63(\mathrm{~m}, 4 \mathrm{H}, \mathrm{F}$-aniline), $5.80(\mathrm{~s}, 2 \mathrm{H}, \mathrm{CS}-\mathrm{OH}), 5.54(\mathrm{~d}, 1 \mathrm{H}, \mathrm{CH}, J=15 \mathrm{~Hz}), 4.85-3.82$ $(\mathrm{m}, 5 \mathrm{H}, \mathrm{CS}-\mathrm{H}), 3.85\left(\mathrm{~s}, 3 \mathrm{H}, \mathrm{OCH}_{3}\right), 3.59(\mathrm{~d}, 2 \mathrm{H}, \mathrm{CS}, J=10 \mathrm{~Hz}), 3.30(\mathrm{~s}, 6 \mathrm{H}, \mathrm{CS}) .{ }^{13} \mathrm{C}$ NMR $\left(\mathrm{CDCl}_{3}\right) \delta(\mathrm{ppm}): 160.6,130.2,124.3,114.5\left(6 \mathrm{C}, \mathrm{OCH}_{3}-\mathrm{Ph}\right), 157.0,155.4,142.5,115.9$ (6C, F-Ph- $\left.\mathrm{NH}_{2}\right), 152.5,124.6,121.7,121.3$ (12C, PO-(Ph) $)$, 102.7, 85.5, 76.3, 73.1, 67.4, 60.1, 59.8, $55.2(8 \mathrm{C}, \mathrm{CS}), 75.0(1 \mathrm{C}, \mathrm{CH}), 55.4\left(1 \mathrm{C}, \mathrm{OCH}_{3}\right)$. EI-MS (relative intensity \%): $m / z 654.24\left(\mathrm{M}^{+}\right.$, 23\%). Elemental analysis: Anal. $\mathrm{C}_{34} \mathrm{H}_{38} \mathrm{FN}_{2} \mathrm{O}_{8} \mathrm{P}: \mathrm{C}, 62.57 ; \mathrm{H}, 5.87$; N, 4.29; Found, C, 62.59; $\mathrm{H}, 5.89$; N, 4.30 .

DiphenylP-((1)-(4-(dimethylamino)phenyl)(((2,3,6)-4-hydroxy-6-(hydroxymethyl)-2,5 -dimethoxytetrahydro-2H-pyran-3-yl)amino)methyl)-N-(4fluorophenyl)phosphonimidate (1e) White solid; yield 80\%; mp $153^{\circ} \mathrm{C}$; IR ( $\left.\mathrm{kBr}\right)\left(\mathrm{cm}^{-1}\right) ; 3430(\mathrm{OH}$, str), 3227 (NH-str), 3034 (CH-str Ar ring), 2936 (CH, str), 1382 (N-C, str), 1176 (P=N), 900 (P-O-C, str). ${ }^{1} \mathrm{H}$ NMR $\left(\mathrm{CDCl}_{3}\right), \delta(\mathrm{ppm}): 9.28(\mathrm{~s}, 1 \mathrm{H}, \mathrm{NH}), 7.68\left(\mathrm{~m}, 4 \mathrm{H}, \mathrm{N}\left(\mathrm{CH}_{3}\right)_{2}-\mathrm{Ph}\right), 7.20-7.05\left(\mathrm{~m}, 10 \mathrm{H}, \mathrm{PO}-(\mathrm{Ph})_{3}\right)$, 6.85-6.61 (m, 4H, F-aniline), $5.82(\mathrm{~s}, 2 \mathrm{H}, \mathrm{CS}-\mathrm{OH}), 5.53(\mathrm{~d}, 1 \mathrm{H}, \mathrm{CH}, J=15 \mathrm{~Hz}), 4.83(\mathrm{~m}, 5 \mathrm{H}$, CS-H), $3.59(\mathrm{~d}, 2 \mathrm{H}, \mathrm{CS}, J=10 \mathrm{~Hz}), 3.30(\mathrm{~s}, 6 \mathrm{H}, \mathrm{CS}), 3.07\left(\mathrm{~s}, 3 \mathrm{H}, \mathrm{N}\left(\mathrm{CH}_{3}\right)_{2}\right) \cdot{ }^{13} \mathrm{C} \mathrm{NMR}\left(\mathrm{CDCl}_{3}\right)$ $\delta$ (ppm):157.0, 155.4, 142.5, 115.9 (6C, F-Ph- $\left.\mathrm{NH}_{2}\right), 152.5,124.6,121.7,121.3$ (12C, PO-(Ph) 3 ), 151.6, 128.9, 120.8, 111.6, $20.4\left(8 \mathrm{C}, \mathrm{N}\left(\mathrm{CH}_{3}\right)_{2}-\mathrm{Ph}\right), 102.0,85.2,76.4,73.5,67.1,60.4,59.7,55.1$ (8C, CS), $75.4(1 \mathrm{C}, \mathrm{CH})$. EI-MS (relative intensity \%): $m / z 667.27\left(\mathrm{M}^{+}, 21 \%\right)$. Elemental analysis: Anal. $\mathrm{C}_{35} \mathrm{H}_{41} \mathrm{FN}_{3} \mathrm{O}_{7} \mathrm{P}: \mathrm{C}, 63.15 ; \mathrm{H}, 6.21 ; \mathrm{N}, 6.33$; Found, $\mathrm{C}, 63.17 ; \mathrm{H}, 6.22 ; \mathrm{N}, 6.35$.

DiphenylN-(4-fluorophenyl)-P-((1)-(((2,3,6)-4-hydroxy-6-(hydroxymethyl)-2,5dimethoxytetrahydro-2H-pyran-3-yl)amino)(3-nitrophenyl)methyl)phosphonimidate (1f) Light yellow solid; yield 92\%; mp $132{ }^{\circ} \mathrm{C}$; IR $(\mathrm{kBr})\left(\mathrm{cm}^{-1}\right)$; $3435(\mathrm{OH}$, str), 3226 (NH-str), 3034 (CH-str Ar ring), 2937 (CH, str), 1550 (N-O, str), 1382 (N-C, str), 1175 (P=N), 903 
(P-O-C, str). ${ }^{1} \mathrm{H}$ NMR $\left(\mathrm{CDCl}_{3}\right), \delta(\mathrm{ppm}): 9.32(\mathrm{~s}, 1 \mathrm{H}, \mathrm{NH}), 8.50-8.30\left(\mathrm{~m}, 4 \mathrm{H}, \mathrm{NO}_{2}-\mathrm{Ph}\right)$, 7.18-6.92 (m, 10H, PO-(Ph) $\left.)_{3}\right), 6.83-6.60(\mathrm{~m}, 4 \mathrm{H}, \mathrm{F}-$ aniline), 5.81 (s, 2H, CS-OH), $5.58(\mathrm{~d}, 1 \mathrm{H}$, $\mathrm{CH}, J=15 \mathrm{~Hz}), 4.82(\mathrm{~m}, 5 \mathrm{H}, \mathrm{CS}-\mathrm{H}), 3.54(\mathrm{~d}, 2 \mathrm{H}, \mathrm{CS}, J=10 \mathrm{~Hz}), 3.32(\mathrm{~s}, 6 \mathrm{H}, \mathrm{CS}) .{ }^{13} \mathrm{C}$ NMR $\left(\mathrm{CDCl}_{3}\right) \delta$ (ppm):157.2, 155.6, 142.4, 115.7 (6C, F-Ph-NH $\left.)_{2}\right), 152.3,124.4,121.6,121.2(12 \mathrm{C}$, $\left.\mathrm{PO}-(\mathrm{Ph})_{3}\right), 148.3,136.1,135.3,130.1,129.5,123.6$ (6C, $\left.\mathrm{NO}_{2}-\mathrm{Ph}\right), 102.1,85.3,76.2,73.4,67.3$, 60.5, 59.6, 55.2 (8C, CS), $75.8(1 \mathrm{C}, \mathrm{CH})$. EI-MS (relative intensity \%): $\mathrm{m} / z 669.22\left(\mathrm{M}^{+}, 20 \%\right)$. Elemental analysis: Anal. $\mathrm{C}_{33} \mathrm{H}_{35} \mathrm{FN}_{3} \mathrm{O}_{9} \mathrm{P}: \mathrm{C}, 59.37 ; \mathrm{H}, 5.28 ; \mathrm{N}, 6.29$; Found, $\mathrm{C}, 59.38 ; \mathrm{H}, 5.29$; $\mathrm{N}, 6.30$.

DiphenylN-(4-fluorophenyl)-P-((1)-(((2,3,6)-4-hydroxy-6-(hydroxymethyl)2,5-dimethoxytetrahydro-2H-pyran-3-yl)amino)(2-hydroxyphenyl)methyl)phosphonimidate (1g) White solid; yield 73\%; mp $160^{\circ} \mathrm{C}$; IR (kBr) $\left(\mathrm{cm}^{-1}\right)$; 3407 (OH, str), 3225 (NH-str), 3034 (CH-str Ar ring), 2934 (CH, str), 1382 (N-C, str), 1174 (P=N), 907 (P-O-C, str). ${ }^{1} \mathrm{H}$ NMR $\left(\mathrm{CDCl}_{3}\right), \delta(\mathrm{ppm}): 9.30(\mathrm{~s}, 1 \mathrm{H}, \mathrm{NH}), 7.72-7.21(\mathrm{~m}, 4 \mathrm{H}, \mathrm{OH}-\mathrm{Ph}), 7.16-6.94\left(\mathrm{~m}, 10 \mathrm{H}, \mathrm{PO}-(\mathrm{Ph})_{3}\right)$, 6.80-6.62 (m, 4H, F-aniline), $5.80(\mathrm{~s}, 2 \mathrm{H}, \mathrm{CS}-\mathrm{OH}), 5.59(\mathrm{~d}, 1 \mathrm{H}, \mathrm{CH}, J=15 \mathrm{~Hz}), 5.35(\mathrm{~s}, 1 \mathrm{H}$, OH-Ph), 4.83-3.98 (m, 5H, CS-H), $3.52(\mathrm{~d}, 2 \mathrm{H}, \mathrm{CS}, J=10 \mathrm{~Hz}), 3.30(\mathrm{~s}, 6 \mathrm{H}, \mathrm{CS}) .{ }^{13} \mathrm{C}$ NMR $\left(\mathrm{CDCl}_{3}\right) \delta$ (ppm): 161.8, 135.9, 131.4, 122.0, 121.5, 118.4 (6C, OH-Ph), 157.3, 155.5, 142.6, 115.3 (6C, F-Ph-NH ${ }_{2}$ ), 152.4, 124.2, 121.5, 121.3 (12C, PO-(Ph) $\left.)_{3}\right), 102.2$, 85.4, 76.5, 73.7, 67.8, 60.6, 59.4, 55.3 (8C, CS), $75.6(1 \mathrm{C}, \mathrm{CH})$. EI-MS (relative intensity \%): $m / z 640.23\left(\mathrm{M}^{+}, 22 \%\right)$. Elemental analysis: Anal. $\mathrm{C}_{33} \mathrm{H}_{36} \mathrm{FN}_{2} \mathrm{O}_{8} \mathrm{P}: \mathrm{C}, 62.06 ; \mathrm{H}, 5.68 ; \mathrm{N}$, 4.39; Found, $\mathrm{C}, 62.07 ; \mathrm{H}, 5.69$; $\mathrm{N}, 4.40$.

DiphenylN-(4-fluorophenyl)-P-((1)-(4-hydroxy-3-methoxyphenyl)(((2,3,6)-4-hydroxy6-(hydroxylmethyl)-2,5-dimethoxytetrahydro-2H-pyran-3-yl)amino)methyl) phosphonimidate (1h) Light yellow solid; yield 86\%; mp $140{ }^{\circ} \mathrm{C}$; IR (kBr) ( $\left.\mathrm{cm}^{-1}\right) ; 3404(\mathrm{OH}, \mathrm{str}), 3220$ (NH-str), 3034 (CH-str Ar ring), 2932 (CH, str), 1382 (N-C, str), 1175 (P=N), 905 (P-O-C, str). ${ }^{1} \mathrm{H} \mathrm{NMR}\left(\mathrm{CDCl}_{3}\right), \delta(\mathrm{ppm}): 9.79(\mathrm{~s}, 1 \mathrm{H}, \mathrm{OH}), 9.28(\mathrm{~s}, 1 \mathrm{H}, \mathrm{NH}), 7.42-6.75(\mathrm{~m}, 3 \mathrm{H}$, vanillin), 7.14-7.06 (m, 10H, PO-(Ph) 3$), 6.82-6.65(\mathrm{~m}, 4 \mathrm{H}, \mathrm{F}$-aniline), $5.83(\mathrm{~s}, 2 \mathrm{H}, \mathrm{CS}-\mathrm{OH}), 5.57(\mathrm{~d}, 1 \mathrm{H}$, $\mathrm{CH}, J=15 \mathrm{~Hz}), 4.70(\mathrm{~m}, 5 \mathrm{H}, \mathrm{CS}-\mathrm{H}), 3.84\left(\mathrm{~s}, 3 \mathrm{H}, \mathrm{OCH}_{3}\right), 3.55(\mathrm{~d}, 2 \mathrm{H}, \mathrm{CS}, J=10 \mathrm{~Hz}), 3.34(\mathrm{~s}$, $6 \mathrm{H}, \mathrm{CS}) \cdot{ }^{13} \mathrm{C}$ NMR $\left(\mathrm{CDCl}_{3}\right) \delta$ (ppm): 157.3, 155.5, 142.6, 115.3 (6C, F-Ph-NH $\left.\mathrm{NH}_{2}\right), 154.5,149.6$, 130.4, 125.2, 117.4, 110.5 (6C, vanillin), 152.6, 124.3, 121.2, 121.4 (12C, PO-(Ph) 3 ), 102.5, 85.3, 76.6, 73.8, 67.4, 60.5, 59.1, 55.2 (8C, CS), $75.3(1 \mathrm{C}, \mathrm{CH}), 56.2\left(1 \mathrm{C}, \mathrm{OCH}_{3}\right)$. EI-MS (relative intensity \%): $m / z 670.24\left(\mathrm{M}^{+}, 19 \%\right)$. Elemental analysis: Anal. $\mathrm{C}_{34} \mathrm{H}_{38} \mathrm{FN}_{2} \mathrm{O}_{9} \mathrm{P}: \mathrm{C}, 51.07 ; \mathrm{H}$, 5.73; N, 4.19; Found, C, 51.09; H, 5.74; N, 4.20.

DiphenylN-(4-fluorophenyl)-P-((1)-(((2,3,6)-4-hydroxy-6-(hydroxymethyl)2,5-dimethoxytetrahydro-2H-pyran-3-yl)amino)-3-phenylallyl)phosphonimidate (1i) White solid; yield 79\%; mp $165^{\circ} \mathrm{C}$; IR ( $\left.\mathrm{kBr}\right)\left(\mathrm{cm}^{-1}\right)$; $3445(\mathrm{OH}$, str), $3223(\mathrm{NH}-\mathrm{str}), 3034(\mathrm{CH}-\mathrm{str}$ Ar ring), $2936\left(\mathrm{CH}\right.$, str), $1383\left(\mathrm{~N}-\mathrm{C}\right.$, str), $1172(\mathrm{P}=\mathrm{N}), 896$ (P-O-C, str). ${ }^{1} \mathrm{H}$ NMR $\left(\mathrm{CDCl}_{3}\right), \delta$ (ppm): $9.29(\mathrm{~s}, 1 \mathrm{H}, \mathrm{NH}), 8.13-7.60(\mathrm{~m}, 7 \mathrm{H}, \mathrm{Ph}), 7.12-6.94(\mathrm{~m}, 10 \mathrm{H}, \mathrm{PO}-(\mathrm{Ph}) 3), 6.84-6.63(\mathrm{~m}$, $4 \mathrm{H}, \mathrm{F}$-aniline), $5.85(\mathrm{~s}, 2 \mathrm{H}, \mathrm{CS}-\mathrm{OH}), 5.60(\mathrm{~d}, 1 \mathrm{H}, \mathrm{CH}, J=17 \mathrm{~Hz}), 4.66(\mathrm{~m}, 5 \mathrm{H}, \mathrm{CS}-\mathrm{H}), 3.58(\mathrm{~d}$, $2 \mathrm{H}, \mathrm{CS}, J=10 \mathrm{~Hz}), 3.30(\mathrm{~s}, 6 \mathrm{H}, \mathrm{CS}) .{ }^{13} \mathrm{C}$ NMR $\left(\mathrm{CDCl}_{3}\right) \delta(\mathrm{ppm}): 157.5,155.3,142.7,115.2(6 \mathrm{C}$, F-Ph- $\left.\mathrm{NH}_{2}\right), 152.6,135.2,128.9,128.6,128.3,127.9$ (8C, Ph), 152.2, 124.6, 121.7, 121.5 (12C, PO-(Ph) 3 ), 102.7, 85.5, 76.3, 73.6, 67.2, 60.3, 59.8, 55.4 (8C, CS), 75.4 (1C, CH). EI-MS (relative intensity \%): $m / z 650.25\left(\mathrm{M}^{+}, 20 \%\right)$. Elemental analysis: Anal. $\mathrm{C}_{35} \mathrm{H}_{38} \mathrm{FN}_{2} \mathrm{O}_{7} \mathrm{P}: \mathrm{C}, 64.81 ; \mathrm{H}$, 5.90; N, 4.32; Found, C, 64.83; H, 5.92; N, 4.34.

DiphenylN-(4-fluorophenyl)-P-((1)-furan-2-yl(((2,3,6)-4-hydroxy-6(hydroxylmethyl)2,5-dimethoxytetrahydro-2H-pyran-3-yl)amino)methyl)phosphonimidate(1j) White solid; yield $80 \%$; mp $143^{\circ} \mathrm{C}$; IR ( $\left.\mathrm{kBr}\right)\left(\mathrm{cm}^{-1}\right) ; 3440(\mathrm{OH}, \mathrm{str}), 3230(\mathrm{NH}-\mathrm{str}), 3036(\mathrm{CH}-\mathrm{str} \mathrm{Ar}$ ring), 2932 (CH, str), 1382 (N-C, str), 1175 (P=N), 900 (P-O-C, str). ${ }^{1} \mathrm{H}$ NMR $\left(\mathrm{CDCl}_{3}\right), \delta$ (ppm):9.29 (s, 1H, NH), 8.09-7.47 (m, 3H, furfural), 7.10-6.92 (m, 10H, PO- $\left.(\mathrm{Ph})_{3}\right), 6.82-6.64(\mathrm{~m}, 4 \mathrm{H}$, F-aniline), $5.82(\mathrm{~s}, 2 \mathrm{H}, \mathrm{CS}-\mathrm{OH}), 5.62(\mathrm{~d}, 1 \mathrm{H}, \mathrm{CH}, J=17 \mathrm{~Hz}), 4.60(\mathrm{~m}, 5 \mathrm{H}, \mathrm{CS}-\mathrm{H}), 3.60(\mathrm{~d}, 2 \mathrm{H}$, $\mathrm{CS}, J=10 \mathrm{~Hz}), 3.33(\mathrm{~s}, 6 \mathrm{H}, \mathrm{CS}) .{ }^{13} \mathrm{C} \mathrm{NMR}\left(\mathrm{CDCl}_{3}\right) \delta(\mathrm{ppm}): 157.5,155.3,142.7,115.2(6 \mathrm{C}$, F-Ph-NH ${ }_{2}$ ), 153.3, 147.2, 121.5, 112.8 (4C, furfural), 152.4, 124.2, 121.5, 121.7 (12C, PO-(Ph) 3 ), 102.6, 85.4, 76.7, 73.2, 67.5, 60.8, 59.1, 55.3 (8C, CS), 75.2 (1C, CH). EI-MS (relative intensity $\%): m / z 614.21\left(\mathrm{M}^{+}, 24 \%\right)$. Elemental analysis: Anal. $\mathrm{C}_{31} \mathrm{H}_{34} \mathrm{FN}_{2} \mathrm{O}_{3} \mathrm{P}: \mathrm{C}, 60.78 ; \mathrm{H}, 5.59 ; \mathrm{N}$, 4.57; Found, C, 60.80; H, 5.61; N, 4.58. 


\subsubsection{Cytotoxic Activity}

The newly synthesized compounds (1a-1j) were screened for their cytotoxic activity according to a procedure described in previous literature [33,34]. Three cancer cell lines were treated with these compoundsat one primary cytotoxic assay dose of $100 \mu \mathrm{M}$ for $48 \mathrm{~h}$ (MTTanticancer assay). Doxorubicin was used as a standard. The two cell lines used in the present investigation were HepG2 (liver) and MCF-7(breast). In the current protocol, all cell lines were pre-incubated on a microtiter plate. The results of each test were reported as the growth percentage of treated cells compared to untreated control cells (Table 1). The screening results were expressed in terms of the growth inhibitor concentration $\left(\mathrm{GI}_{50}\right)$, total growth of inhibition (TGI), and lethal concentration $\left(\mathrm{LC}_{50}\right)$. A $0.1 \mathrm{~mL}$ aliquot ofthecell suspension $\left(5 \times 10^{4}\right.$ cells $\left./ 100 \mu \mathrm{L}\right)$ and $0.1 \mathrm{~mL}$ ofthetest solution $(6.25-100 \mu \mathrm{g}$ in $1 \%$ DMSO, the final DMSO concentration in media less than $1 \%$ ) were added to the wells, with the plates kept in an incubator $\left(5 \% \mathrm{CO}_{2}\right)$ at $37^{\circ} \mathrm{C}$ for $72 \mathrm{~h}$. The results were analyzed using the statistical software SPSS version 16.0. Manufacturers and suppliers of SPSS software company IBM located in USA, New York, Armonk.

Table 1. Cytotoxic effect of compounds $(\mu \mathrm{M}) \mathbf{1 a}-\mathbf{1 j}$.

\begin{tabular}{ccccccc}
\hline \multirow{2}{*}{ Compounds } & \multicolumn{3}{c}{ HepG2 } & \multicolumn{3}{c}{ MCF-7 } \\
\cline { 2 - 7 } & $\begin{array}{c}\mathbf{G I}_{\mathbf{5 0}} \\
(\boldsymbol{\mu M})\end{array}$ & $\begin{array}{c}\text { TGI } \\
(\boldsymbol{\mu M})\end{array}$ & $\begin{array}{c}\mathbf{L C}_{\mathbf{5 0}} \\
(\boldsymbol{\mu M})\end{array}$ & $\begin{array}{c}\mathbf{G I}_{\mathbf{5 0}} \\
(\boldsymbol{\mu M})\end{array}$ & $\begin{array}{c}\text { TGI } \\
(\boldsymbol{\mu M})\end{array}$ & $\begin{array}{c}\mathbf{L C}_{\mathbf{5 0}} \\
(\boldsymbol{\mu M})\end{array}$ \\
\hline 1a & $5.1 \pm 0.39$ & $10.1 \pm 0.26$ & $19.2 \pm 0.15$ & $2.9 \pm 0.21$ & $4.2 \pm 0.20$ & $10.2 \pm 0.02$ \\
\hline $\mathbf{1 b}$ & $3.3 \pm 0.24$ & $7.2 \pm 0.54$ & $16.2 \pm 0.38$ & $0.23 \pm 0.30$ & $5.0 \pm 0.74$ & $18.2 \pm 0.12$ \\
\hline $\mathbf{1 c}$ & $0.07 \pm 0.09$ & $3.1 \pm 0.82$ & $6.2 \pm 0.08$ & $0.01 \pm 0.28$ & $0.18 \pm 0.31$ & $0.20 \pm 0.05$ \\
\hline $\mathbf{1 d}$ & $0.09 \pm 0.14$ & $0.12 \pm 0.20$ & $0.25 \pm 0.18$ & $5.9 \pm 0.14$ & $10.5 \pm 0.48$ & $19.2 \pm 0.11$ \\
\hline $\mathbf{1 e}$ & $4.9 \pm 0.20$ & $9.2 \pm 0.23$ & $17.2 \pm 0.12$ & $1.9 \pm 0.98$ & $7.0 \pm 0.34$ & $14.2 \pm 0.16$ \\
\hline $\mathbf{1 f}$ & $3.1 \pm 0.32$ & $6.9 \pm 0.21$ & $12.2 \pm 0.10$ & $2.2 \pm 0.71$ & $4.3 \pm 0.13$ & $8.2 \pm 0.10$ \\
\hline $\mathbf{1 g}$ & $1.4 \pm 0.11$ & $2.8 \pm 0.41$ & $18.2 \pm 0.12$ & $1.5 \pm 0.38$ & $6.3 \pm 0.13$ & $16.2 \pm 0.65$ \\
\hline $\mathbf{1 h}$ & $0.02 \pm 0.20$ & $0.14 \pm 0.85$ & $0.57 \pm 0.12$ & $0.04 \pm 0.29$ & $0.31 \pm 0.62$ & $0.75 \pm 0.12$ \\
\hline $\mathbf{1 i}$ & $7.9 \pm 0.33$ & $14.9 \pm 0.45$ & $26.9 \pm 0.91$ & $2.7 \pm 0.27$ & $7.9 \pm 0.17$ & $12.3 \pm 0.19$ \\
\hline $\mathbf{1 j}$ & $4.8 \pm 0.21$ & $8.8 \pm 0.87$ & $16.9 \pm 0.57$ & $4.2 \pm 0.39$ & $8.4 \pm 0.84$ & $20.3 \pm 0.98$ \\
\hline Doxorubicin & $0.01 \pm 0.20$ & $0.13 \pm 0.14$ & $0.58 \pm 0.08$ & $0.02 \pm 0.52$ & $0.21 \pm 0.84$ & $0.74 \pm 0.20$ \\
\hline
\end{tabular}

Data represent the mean \pm standard deviation (SD) of the mean values of three separate experiments.

\subsubsection{Molecular Docking}

Molecular docking tests are regularly used to analyze binding interactions. The relationships of between the target protein (PDB ID: 6LU7) and the synthesized chitosan derivatives 1a-1j were determined with AutodockVina 1.1 [35]. The crystal structure SARS coronavirus core protease (PDB ID: 6LU7) was obtained from the Protein Data Bank (http:/www.rcsb.org (accessed on 5 September 2020)). ChemDraw Ultra 12.0 and Chem3D Pro 12.0 programming suites were utilized to sketch the 3D structures of the ligand molecules and energyminimization. AutoDock Tools 1.5.6 was utilized to fabricate the AutodockVina input contents. In manufacturing the co-crystallized inhibitor molecule using the discovery studio program, $\alpha$-ketoamide was used to identify the binding pocket. The amino acid residues Thr24, Thr26, Phe140, Asn142, Gly143, Cys145, His163, His164, Glu166, and His172were present in the binding pocket. The SARS COVID-19 main protease quest grid was recognized as center $\mathrm{x}$ : -10.656 , center $\mathrm{y}$ : 17.223 , center $\mathrm{z}$ : 67.024 in dimension x: 20, y: 20, and z: 20 in $1.0 \AA$ A spacing. The significance of completeness was set to 8 . The extra limitations were fixed and not indicated by default for Autodock Vina. Thecompound that consumed the smallest binding affinity value is achieved the highest score, the implications of which were visually examined with Discovery studio 2019. 


\subsubsection{Structure Activity Relationship}

A structure-activity relationship analysis (SAR) can be used to investigate the relationship between the chemical structure of an active molecule and its biological activity in a specific assay system. SAR analysis makes it possible to identify the chemical group/atom that plays a critical role in modulating the cytotoxic activity of compounds in the specific system. Based on the cytotoxic activity results of thederivatives preliminary SARs could be evaluated. The data of the selected chitosan derivatives (1a-1j) evidenced that compound 1c is highly active $\left(\mathrm{GI}_{50} 0.01 \mu \mathrm{M}\right)$ against the MCF-7 cancer cell linecompared with control Doxorubicin. Compound $1 \mathrm{c}$ docking score $-7.9 \mathrm{kcal} / \mathrm{mol}$. Figure 4 indicates highly active compound in cytotoxic activity and molecular docking analysis.

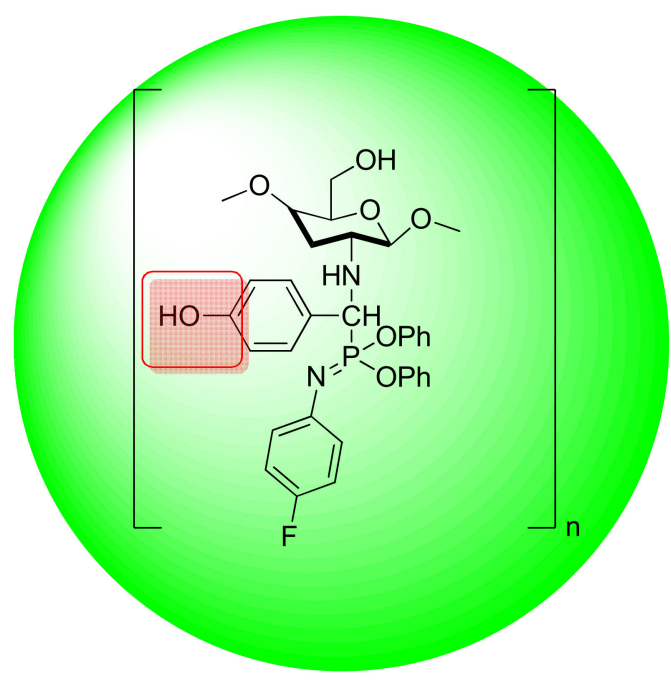

Figure 4. Highly active compound structure.

Due to the presence of $\beta$-chitosan beingfused to a hydroxy benzaldehyde, it was found that the compound acquires a high cytotoxic activity against cancer cell lines.

The reason would be the presence of electron releasing hydroxyl group on phenyl ring attached with the $\beta$-chitosan skeleton. The rest of the compounds showed weak cytotoxic activity against all the tested cancer cell lines. Moreover, from the docking results, it can be assumed that the docking score for $\beta$-chitosan derivatives (1a-1j) has an acceptable range.

\section{Result and Discussion}

The chitosan derivatives of compound $\mathbf{1}$ were prepared following a method found in the literature [36]. Synthesis of amino phosphate chitosan involved Schiff base formation and fluoro aniline was added to $\beta$-chitosan (Mwt.577) (1a-1j) by the procedure described in [37]. The amino group present in $\beta$-chitosan (C2-Position) are significant because they are nucleophilic and eagerly react with electrophilic reagents. Because of this, biologically active moieties were grafted into the amino group of chitosan. Compound 1 reacted with 4fluoroaniline, $\mathrm{NaOH}$, and ethanol added at high temperature and underwent a dehydration reaction to obtain Compound 1a as a white solid. The reaction was monitored by thin layer chromatography (TLC). After completion of the reaction, the crude product was recrystallized by ethanol. The above procedure was followed for other (1b-1j) synthesized chitosan derivatives. The synthesized compounds were confirmed by analytical technique, such as FT-IR, ${ }^{1} \mathrm{H}$ NMR and ${ }^{13} \mathrm{C}$ NMR as seen in Supplementary Materials (Figures S1-S3). The FT-IR spectra exhibited absorption bands (1a-1j) at 895, 1170, 1383, 3045, 3250, and $3450 \mathrm{~cm}^{-1}$, confirming $\mathrm{P}-\mathrm{O}-\mathrm{C}, \mathrm{P}=\mathrm{N}, \mathrm{N}-\mathrm{C}, \mathrm{Ar}-\mathrm{H}, \mathrm{NH}$, and $\mathrm{OH}$ functional groups. The ${ }^{1} \mathrm{H}$ NMR spectra (1a-1j) showed chemical shift values at $\delta 10.60,9.34-7.95,8.50-6.64,7.28-6.96$, 6.87-6.60, 5.55, and $3.85 \mathrm{ppm}$, confirmingOH, NH, P-O-Ph, $\mathrm{F}_{-} \mathrm{NH}_{2}, \mathrm{CH}$, and $\mathrm{OCH}_{3}$ protons, respectively. The ${ }^{13} \mathrm{C}$ NMR showed (1b-1j) signals at157.7-115.3, 152.7-121.3, 75.3, and 
$56.2 \mathrm{ppm}$, confirming the $\mathrm{F}-\mathrm{NH}_{2}, \mathrm{P}-\mathrm{O}-\mathrm{Ph}, \mathrm{CH}$, and $\mathrm{OCH}_{3}$ of carbon atoms. Chitosan derivatives were affirmed by mass spectroscopy and elemental analysis. The synthesis of chitosan derivatives are shown in Scheme 1.

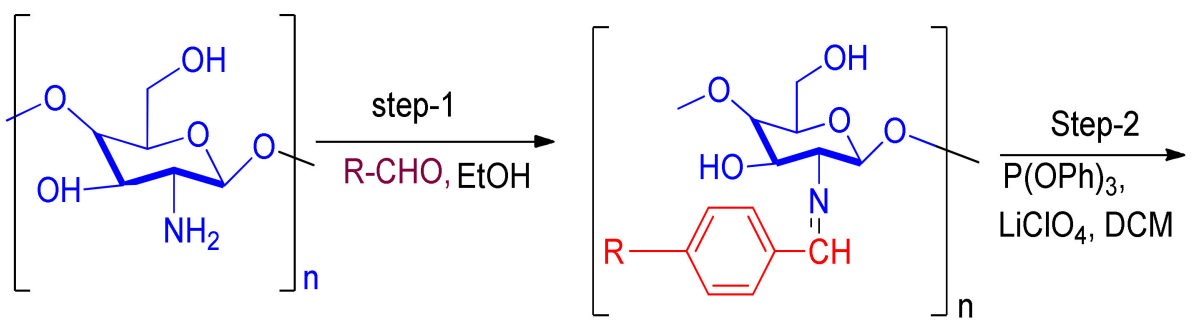<smiles>[R]c1ccc(C(NC(OCCO)C(O)COC)P(=O)(O)Oc2ccccc2)cc1</smiles>

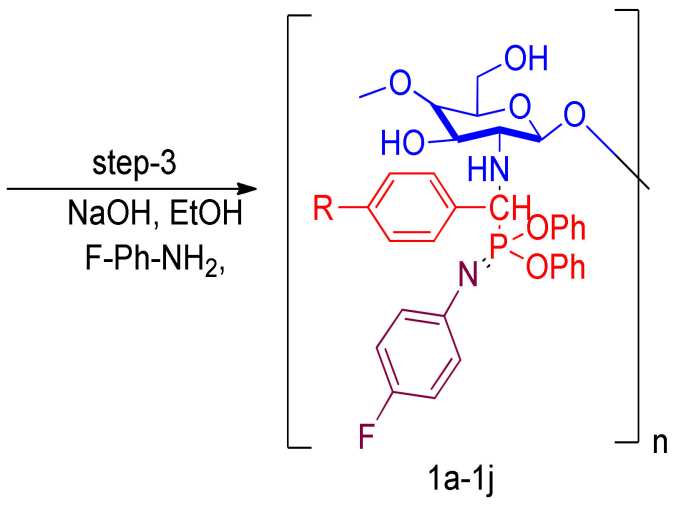

\section{R=F-Ph- $\mathrm{NH}_{2}, 4 \mathrm{ClC}_{6} \mathrm{H}_{4}, 4-\mathrm{OHC}_{6} \mathrm{H}_{4}, 4-\mathrm{CH}_{3} \mathrm{OC}_{6} \mathrm{H}_{4}, 4\left(\mathrm{CH}_{3}\right)_{2} \mathrm{NC}_{6} \mathrm{H}_{4}, 3-$ $\mathrm{NO}_{2} \mathrm{C}_{6} \mathrm{H}_{4}, 2-\mathrm{OHC}_{6} \mathrm{H}_{4}$, Vanillin, $\mathrm{Ph}-\mathrm{CH}=\mathrm{CH}-\mathrm{CHO}$, Furfural}

Scheme 1. Synthesis of chitosan derivatives.

\subsection{XRD (or) X-ray Diffraction Study}

The chitosan and chitosan derivatives were carried out in an X-Ray diffraction study. Chitosan has observed with very broad peaks at $2 \theta=10^{\circ}$ and $2 \theta=20^{\circ}$ Figure 5 [38]. The chitosan derivatives showed two weak peaks at $2 \theta$ of $21^{\circ}$ and $38^{\circ}$ Figure 5. The peak detected for chitosan at $2 \theta=10^{\circ}$ disappeared and the very broad peaks at $20^{\circ}$ became weak in chitosan derivatives, it shows that chitosan has moral compatibility and good formation of porous xerogel network. The XRD result indicates that chitosan derivatives are an amorphous form.

\subsubsection{Thermal Analysis of TGA}

Figure 6 shows TGA thermograms of pure chitosan and chitosan derivatives. The weight loss of pure chitosan occurs in two stages at the range of 50 to $455^{\circ} \mathrm{C}$. The first occurs in the range of $50-100{ }^{\circ} \mathrm{C}$ due to loss of $\mathrm{H}_{2} \mathrm{O}$ molecule with weight loss of $10 \%$. The pure chitosan primary degradation starts at $250{ }^{\circ} \mathrm{C}$, and the entire pure chitosan at $455{ }^{\circ} \mathrm{C}$ with a weight loss of $35 \%$ [38]. TGA of chitosan derivatives displayed two stages of weight loss in Figure 6. Initial weight loss of chitosan derivative starting from $31{ }^{\circ} \mathrm{C}$ to $92{ }^{\circ} \mathrm{C}$ may represent a loss of adsorbed $\mathrm{H}_{2} \mathrm{O}$ molecule. The next (second) stage decomposition occurs in the range of $230-410{ }^{\circ} \mathrm{C}$, due to heat decomposition with a weight loss of $51 \%$. The above result describes the loss of thermal stability for chitosan derivatives compared with pure chitosan. 


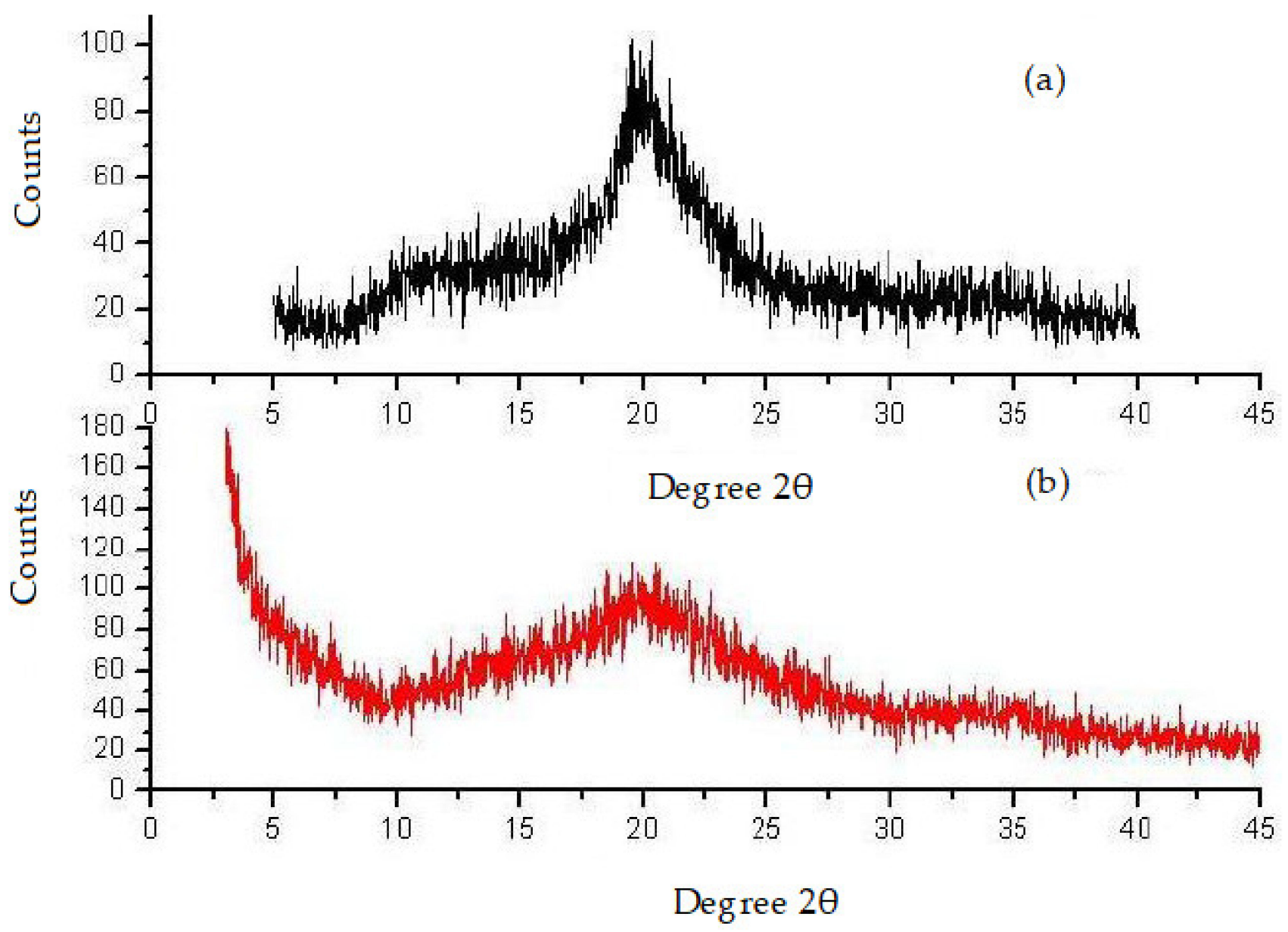

Figure 5. X-ray Diffraction Study of pure chitosan (a) and chitosan derivative (b).

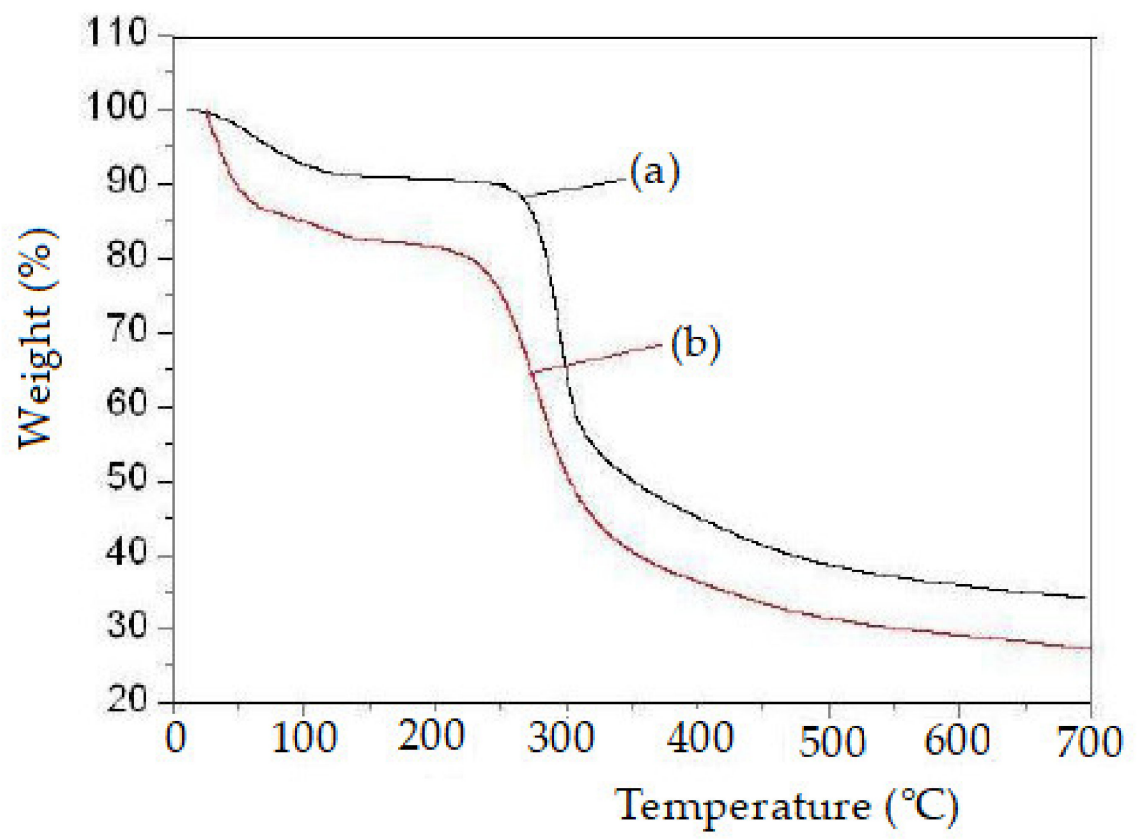

Figure 6. Thermogravimetric analysisof pure chitosan (a) and chitosan derivative (b). 


\subsubsection{Thermal Analysis of DSC}

The DSC thermogram of chitosan derivatives is given in Figure 7. Figure 7 indicates the sharp endothermic peaks at $95{ }^{\circ} \mathrm{C}$ due to the loss of $\mathrm{H}_{2} \mathrm{O}$ molecules. Then the thermal decomposition of the chitosan derivative occurs in one exothermic peak at $298^{\circ} \mathrm{C}$. Finally, the structures of the chitosan chain have changed due to the fluoro aniline (or) triphenylphosphite and also have a reduced ability to crystalize.

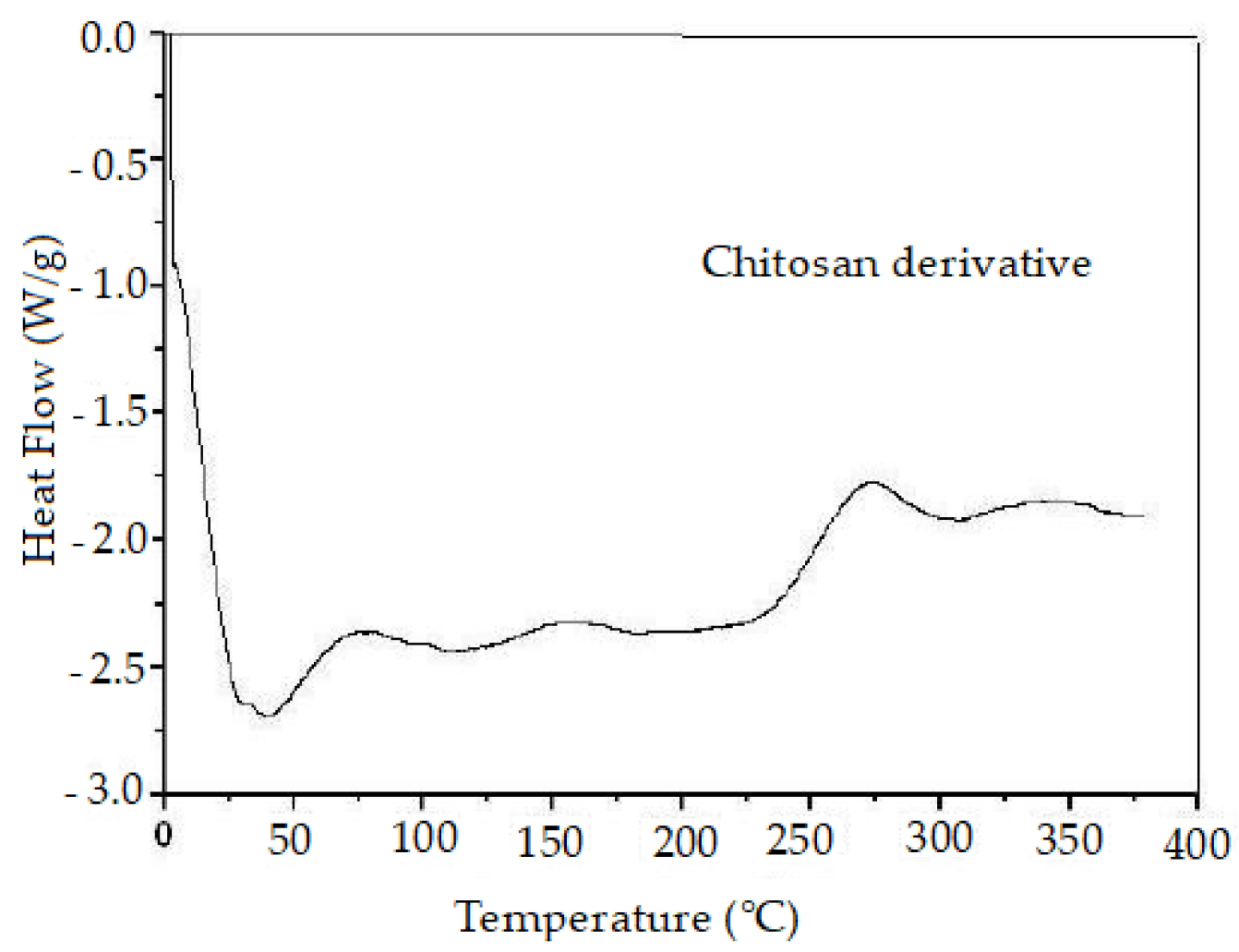

Figure 7. Differential scanning calorimetry of chitosan derivative.

\subsubsection{Cytotoxicity Screening}

The compounds were tested for cytotoxicity against the HepG2 and MCF-7 cancer cell lines. Compound $1 \mathrm{~h}$ showed moderate activity against HepG2 $\left(\mathrm{GI}_{50} 0.02 \mu \mathrm{M}\right)$, and compound $1 \mathrm{c}$ is highly active $\left(\mathrm{GI}_{50} 0.01 \mu \mathrm{M}\right)$ against the MCF-7 cancer cell line. All other compounds exhibited lower activity compared to doxorubicin. Values are given in Table 1.

\subsubsection{Docking Studies}

Docking stimulations were carried out in order to foster understanding of the possible biological activity processes. The compounds (1a-1j) were analyzed by AutodockVina for their docking behavior with the SARS corona virus main protease (PDB ID: 6LU7). The studied inhibitors show negative binding energy. Compound 1c demonstrates a remarkable inhibition ability with a binding energy value of $-7.9 \mathrm{kcal} / \mathrm{mol}$, compared with compounds $1 \mathrm{a}(-7.7 \mathrm{kcal} / \mathrm{mol}), \mathbf{1 b}(-7.3 \mathrm{kcal} / \mathrm{mol}), 1 \mathrm{~d}(-7.8 \mathrm{kcal} / \mathrm{mol}), 1 \mathrm{e}(-7.4 \mathrm{kcal} / \mathrm{mol})$, 1f $(-7.8 \mathrm{kcal} / \mathrm{mol}), 1 \mathrm{~g}(-7.4 \mathrm{kcal} / \mathrm{mol}), 1 \mathrm{~h}(-7.2 \mathrm{kcal} / \mathrm{mol}), 1 \mathrm{i}(-7.8 \mathrm{kcal} / \mathrm{mol})$, and $1 \mathrm{j}$ $(-7.5 \mathrm{kcal} / \mathrm{mol})$ in the target protein correspondingly.Hydrogen bonding serves a significant function in ligand binding stability, and the approvable bond gap between $\mathrm{H}$-acceptor and $\mathrm{H}$-donor atoms is less than $3.5 \AA$ [39]. The gap between the hydrogen bound compounds in the target protein $6 \mathrm{LU} 7$ was less than $3.5 \AA$, which means that the protein and ligands were involved in a resilient hydrogen bond interaction. Compound $1 \mathrm{c}$ displays two hydrogen bonding associations with the 6LU7 receptor. The amino acid residues of Glu166 and Gln189 were entangled through the relationship of the hydrogen linkage with 
bond lengths between 2.69 and $1.98 \AA$. The amino acid residues of Leu27, His41, Met49, and Cys145 were in hydrophobic contact. Figure 8 reveals the hydrogen bonding and hydrophobic contacts in the 6LU7 protein of the amino acid residue with compound 1c. The findings reveal that compound 1c has an excellent capacity to inhibit the SARS corona virus main protease (PDB ID: 6LU7) compared with other compounds. The findings are presented in Table 2.

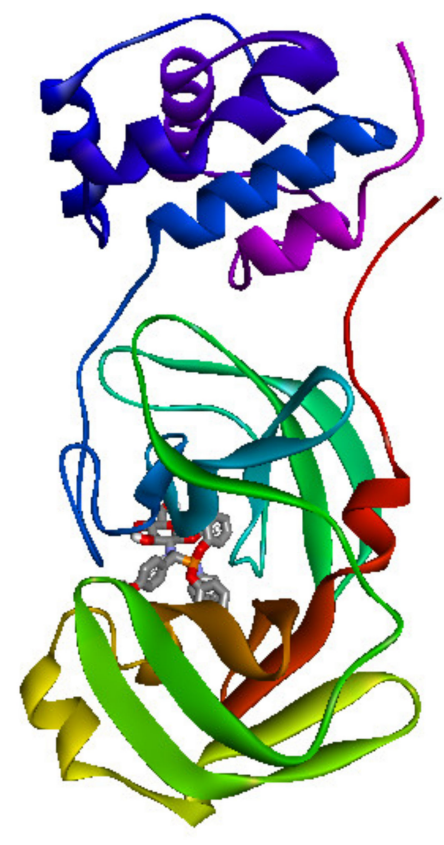

(a)

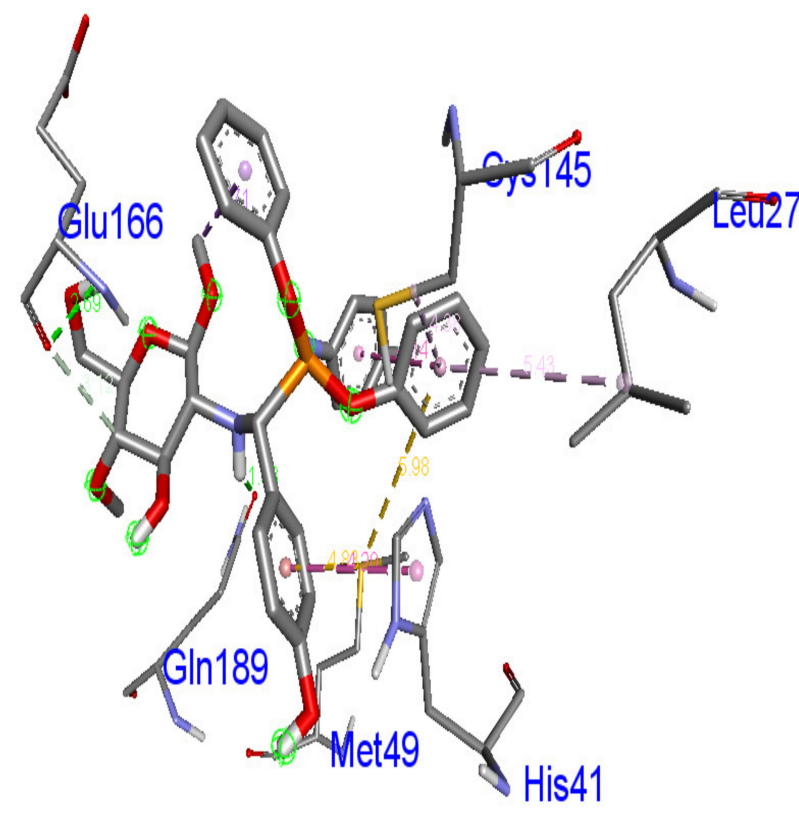

(c)

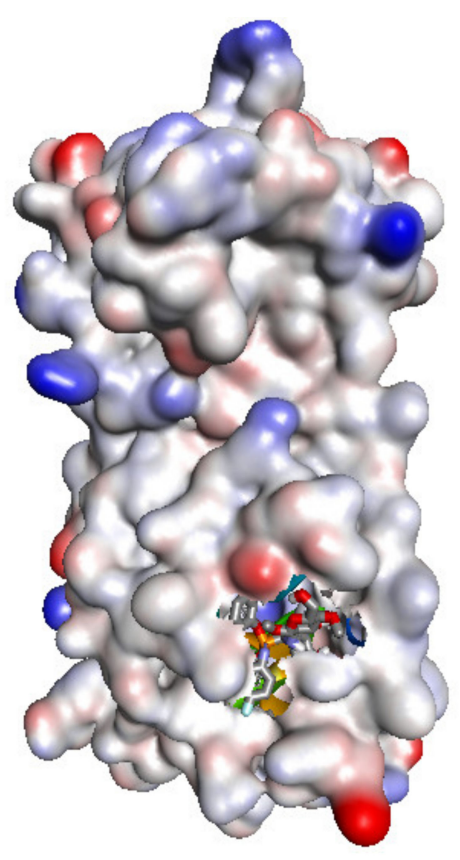

(b)

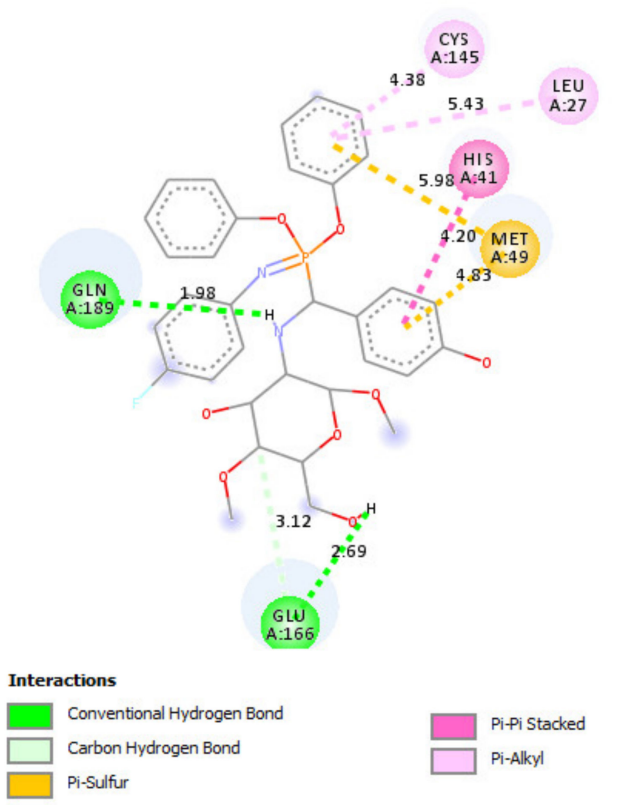

(d)

Figure 8. Helix (a), molecular surface (b), 3D (c), and 2D (d) interaction modes of compound 1c within the binding site of. 6LU7 protein. 
Table 2. Molecular docking interaction of compounds (1a-1j) against protease of SARS coronavirus in complex with $\alpha$-ketoamide (PDB ID: 6LU7).

\begin{tabular}{cccc}
\hline \multirow{2}{*}{ Compounds } & \multicolumn{3}{c}{ Main Protease of SARS Coronavirus (PDB ID: 6LU7) } \\
\cline { 2 - 4 } & Binding Affinity (kcal/mol) & No. of H-Bonds & H-Bonding Residues \\
\hline$(\mathbf{1 a})$ & -7.7 & 2 & Glu166, Gln189 \\
$(\mathbf{1 b})$ & -7.3 & 2 & Gly143, Gln189 \\
$(\mathbf{1 c})$ & -7.9 & 2 & Glu166, Gln189 \\
$(\mathbf{1 d})$ & -7.8 & 2 & Glu166, Gln189 \\
$(\mathbf{1 e})$ & -7.4 & 2 & Asn142, Gln189 \\
$(\mathbf{1 f})$ & -7.8 & 0 & - \\
$(\mathbf{1 g})$ & -7.4 & 1 & Gly143 \\
$(\mathbf{1 h})$ & -7.2 & 2 & Gly143, Gln189 \\
$(\mathbf{1 i})$ & -7.8 & 1 & Asn142, Gln189 \\
$(\mathbf{1 j})$ & -7.5 & 1 & Cys145 \\
\hline
\end{tabular}

\section{Conclusions}

Newly synthesized chitosan analogs (1a-1j) were confirmed by spectroscopic, $\mathrm{X}$ ray diffraction, TGA, and DSC techniques and screened forin-silico molecular docking analysis against the SARS coronavirus main protease (PDB ID: 6LU7). Compound 1c exhibits excellent binding affinity $-7.9 \mathrm{kcal} / \mathrm{mol}$ against the target protein (PDB ID: 6LU7). Compound $1 \mathrm{~h}\left(\mathrm{GI}_{50} 0.02 \mu \mathrm{M}\right)$ is moderately active against the HepG2 cancer cell line, and Compound 1c is highly active $\left(\mathrm{GI}_{50} 0.01 \mu \mathrm{M}\right)$ against the MCF-7 cancer cell line. The synthesized chitosan derivative of compound $1 \mathrm{c}$ is a potential antiviral drug and isconsidered a potential therapeutic molecule for COVID-19.

Supplementary Materials: The following are available online at https:/ /www.mdpi.com/article/10 .3390/polym13071046/s1, Figure S1. FT-IR spectra of compound 1a-1j. Figure S2. 1H-NMR spectra of compound 1a-1j. Figure S3. 13C-NMR spectra of compound 1a-1j.

Author Contributions: P.P.: Preparation of synthetic compounds and chemical data analysis; P.G.: Organic compound preparation; D.A.: Software analysis; S.A.: Financial support; N.S.A.: Data collection; A.I.: Spectral analysis; R.S.: Investigations and manuscript preparation. All authors have read and agreed to the published version of the manuscript.

Funding: This research received no external funding.

Institutional Review Board Statement: Not applicable.

Informed Consent Statement: Not applicable.

Data Availability Statement: Data available in Supplementary Materials.

Acknowledgments: This research work was funded by the Deanship of Scientific Research at Princess Nourah bint Abdulrahman University through the Fast-track Research Funding Program.

Conflicts of Interest: The authors declare no conflict of interest.

\section{References}

1. World Health Organization. Emergencies Preparedness, Response. Severe Acute Respiratory Syndrome (SARS). Available online: https:/ / www.who.int/csr/don/archive/disease/severeacuterespiratorysyndrome/en (accessed on 18 May 2004).

2. World Health Organization. GCM Teleconference-Note for the Records. 10 January 2020. Subject: Pneumonia in Wuhan, China. Available online: https:/ / www.who.int/blueprint/10-01-2020-nfr-gcm.pdf (accessed on 10 January 2021).

3. Talha, K.B. The Russian vaccine for COVID-19. NEWS 2020, 8, e85-e86.

4. So, L.K.; Lau, A.C.W.; Yam, L.Y.C.; Cheung, T.M.T.; Poon, E.; Yung, R.W.H.; Yuen, K.Y. Development of a standard treatment protocol for severe acute respiratory syndrome. Lancet 2003, 361, 1615-1617. [CrossRef]

5. Hon, K.L.E.; Leung, C.W.; Cheng, W.T.F.; Chan, P.K.S.; Chu, W.C.W.; Kwan, Y.W.; Li, A.M.; Fong, N.C.; Ng, P.C.; Chiu, M.C.; et al. Clinical presentations and outcome of severe acute respiratory syndrome in children. Lancet 2003, 361, 1701-1703. [CrossRef]

6. Yamamoto, N.; Yang, R.; Yoshinaka, Y.; Amari, S.; Nakano, T.; Cinatl, J.; Rabenau, H.; Doerr, H.W.; Hunsmann, G.; Otaka, A.; et al. HIV Protease inhibitor nelfinavir inhibits replication of SARS-associated coronavirus. Biochem. Biophys. Res. Commun. 2004, 318, 719-725. [CrossRef] 
7. Anand, K.; Ziebuhr, J.; Wadhwani, P.; Mesters, J.R.; Hilgenfeld, R. Coronavirus Main Proteinase (3CLpro) Structure: Basis for Design of Anti-SARS Drugs. Science 2003, 300, 1763-1767. [CrossRef]

8. Xiaofei, H.; Ronge, X.; Song, L.; Yukun, Q.; Kecheng, L.; Huahua, Y.; Pengcheng, L. The improved antiviral activities of amino modified chitosan derivatives on Newcastle virus. Drug Chem. Toxicol. 2019, 1-6.

9. Chirkov, S.N. The Antiviral Activity of Chitosan (Review). Appl. Biochem. Microbiol. 2002, 38, 1-8, Translated from Prikl Biokhim Mikrobiolo. 2002, 38, 5-13. [CrossRef]

10. Nadia, Q.H.; Mohsin, O.M.; Luqman, E.M. Synthesis and Biological Evaluation of Three New Chitosan Schiff Base Derivatives. ACS Omega 2020, 5, 13948-13954.

11. Blackwell, J. Physical methods for the determination of chitin structure and conformation. Methods Enzymol. 1988, 161, 435-442.

12. Hamed, A.A.; Abdelhamid, I.A.; Saad, G.R.; Elkady, N.A.; Elsabee, M.Z. Synthesis, Characterization and Antimicrobial Activity of a Novel Chitosan Schiff Bases Based on Heterocyclic Moieties. Int.J. Biol. Macromol. 2020, 153, 492-501. [CrossRef] [PubMed]

13. MuhdJulkapli, N.; Akil, H.M.; Ahmad, Z. Preparation, Properties and Applications of Chitosan-Based Biocomposites/Blend Materials: A Review. Compos. Interfaces 2011, 18, 449-507. [CrossRef]

14. Casadidio, C.; Peregrina, D.V.; Gigliobianco, M.R.; Deng, S.; Censi, R.; Di Martino, P. Chitin and Chitosans: Characteristics, EcoFriendly Processes, and Applications in Cosmetic Science. Mar. Drugs 2019, 17, 369. [CrossRef] [PubMed]

15. Matica, M.A.; Aachmann, F.L.; Tøndervik, A.; Sletta, H.; Ostafe, V. Chitosan as a Wound Dressing Starting Material: Antimicrobial Properties and Mode of Action. Int. J. Mol. Sci. 2019, 20, 5889. [CrossRef] [PubMed]

16. Chirkov, S.N.; Surgucheva, N.A.; Gamzazade, A.I.; Abdulabekov, I.M.; Pospeshny, G. Relative efficiency of chitosan derivatives in the inhibition of viral infection of plants. Dokl. Akad. Nauk. 1998, 360, 271-273.

17. Pospieszny, H.; Struszczyk, H.; Cajza, M.; Muzzarelli, R.A.A. Ancona: In Chitin enzymology. Atec. Grottamare. 1996, 2, 385-389.

18. Struszczyk, M.H.; Pospieszny, H.; Schanzenbach, D.; Peter, M.G. In Progress on Chemistry and Application of Chitin and its Derivatives; Struszczyk, H., Ed., Lodz. In Pol. Chitin Soc.; 1998; Volume 5, pp. 71-77.

19. Ying, G.Q.; Xiong, W.Y.; Wang, H.; Sun, Y.; Liu, H.Z. Preparation, water solubility and antioxidant activity of branched-chain chitosan derivatives. Carbohydr. Polym. 2011, 83, 1787-1796. [CrossRef]

20. Chirkov, S.N.; Mikrobiol, P.B. The Antiviral Activity of Chitosan (Review). Prikl. Biokhimiya Mikrobiol. 2002, 38, 5-13.

21. Rhoades, J.; Roller, S. Antimicrobial Actions of Degraded and Native Chitosan against Spoilage Organisms in Laboratory Media and Foods. Appl. Environ. Microbiol. 2000, 66, 80-86. [CrossRef] [PubMed]

22. Kumar, S.; Koh, J. Physiochemical, Optical and Biological Activity of Chitosan-Chromone Derivative for Biomedical Applications. Int. J. Mol. Sci. 2012, 13, 6102-6116. [CrossRef] [PubMed]

23. Hamodrakas, S.J.; Jones, C.W.; Kafatos, F.C. Secondary structure predictions for silkmoth chorion proteins. Biochem. Biophys. Acta (BBA) Protein Struct. Mol. Enzymol. 1982, 700, 42-51. [CrossRef]

24. Nishi, N.; Ebina, A.; Nishimura, S.; Tsutsumi, A.; Hasegawa, O.; Tokura, S. Highly phosphorylated derivatives of chitin, partially deacetylated chitin and chitosan as new functional polymers: Preparation and characterization. Int. J. Biol. Macromol. 1986, 8 , 311-317. [CrossRef]

25. Li, C.; Song, B.; Yan, K.; Xu, G.; Hu, D.; Yang, S.; Jin, L.; Xue, W.; Lu, P. One Pot Synthesis of $\alpha$-Aminophosphonates Containing Bromo and 3,4,5-Trimethoxybenzyl Groupsunder Solvent-freeConditions. Molecules 2007, 12, 163-172. [CrossRef]

26. Prasad, G.S.; Krishna, J.R.; Manjunath, M.; Reddy, O.V.S.; Krishnaiah, M.; Reddy, C.S.; Puranikd, V.G. Synthesis, NMR, X-ray crystallography and bioactivity of some $\alpha$ - Aminophosphonates. Arkivoc 2007, 13, 133-141. [CrossRef]

27. Rao, X.; Song, Z.; He, L. Synthesis and antitumor activity of novel $\alpha$-aminophosphonates from diterpenicdehydroabietylamine. Heteroat. Chem. 2008, 19, 512-516. [CrossRef]

28. Naydenova, E.D.; Todorov, P.T.; Topashka-Ancheva, M.N.; Momekov, G.T.; Yordanova, T.Z.; Konstantinov, S.M.; Troev, K.D. Novel $\mathrm{N}$-(phosphonomethyl) glycine derivatives: Design, characterization and biological activity. Eur. J. Med. Chem. 2008, 43, 1199-1205. [CrossRef] [PubMed]

29. Tusek-Bozic, L.; Juribasic, M.; Traldi, P.; Scarcia, V.; Furlani, A. Synthesis, characterization and antitumor activity of palladum(II) complexes of monoethyl 8-quinolylmethyl phosphonate. Polyhedron 2008, 27, 1317-1328. [CrossRef]

30. Wang, B.; Miao, Z.W.; Wang, J.; Chen, R.Y.; Zhang, X.D. Synthesis and evaluation of novelnaphthoquinone fused cyclic amino alkyl phosphonates and amino alkyl phosphonic monoester. AminoAcids 2008, 35, 463-468.

31. Jiang, M.; Ouyang, H.; Ruan, P.; Zhao, H.; Pi, Z.; Huang, S.; Yi, P.; Crepin, M. Chitosan Derivatives Inhibit Cell Proliferation and Induce Apoptosis in Breast Cancer Cells. Anticancer Res. 2011, 3, 1321-1328.

32. Li, Q.; Li, Q.; Tan, W.; Zhang, J.; Guo, Z. Phenolic-containing chitosan quaternary ammonium derivatives and their significantly enhanced antioxidant and antitumor properties. Carbohydr. Res. 2020, 498, 108169. [CrossRef]

33. Surendra Kumar, R.; Moydeen, M.; Al-Deyab, S.; Aseer, M.; Idhayadhulla, A. Synthesis of new morpholine- connected pyrazolidine derivatives and their antimicrobial, antioxidant, and cytotoxic activities. Bio. Med. Chem. Lett. 2017, 27, 66-71. [CrossRef] [PubMed]

34. Scudiero, D.A.; Shoemaker, R.H.; Paull, K.D.; Monks, A.; Tierney, S. Evaluation of a soluble tetrazolium/formazan assay for cell growth and drug sensitivity in culture using human and other tumor cell lines. Cancer Res. 1988, 48, 4827-4833. [PubMed]

35. Vina, A. Improving the speed and accuracy of docking with a new scoring function, efficient optimization, and multithreading. $J$. Comput. Chem. 2010, 31, 455-461. 
36. El-Refaie, S.K.; Mohamed, M.A.; Khalil, M.S. Synthesis and antimicrobial activity of a-amino phosphonates containing chitosan moiety. Arbian J. Chem. 2015, 8, 427-432.

37. Barbosa, H.F.G.; Attjioui, M.; Ferreira, A.P.G.; Moerschbacher, B.M.; Cavalheiro, É.T.G. New series of metal complexes by amphiphilic biopolymeric Schiff bases from modified chitosans: Preparation, characterization and effect of molecular weight on its biological applications. Int. J. Biol. Macromol. 2020, 145, 417-428. [CrossRef]

38. Kumar, S.; Dutta, J.; Dutta, P.K. Preparation and characterization of N-heterocyclic chitosan derivative based gels for biomedical applications. Int. J. Biol. Macromol. 2009, 45, 330-337. [CrossRef] [PubMed]

39. Taha, M.; Ismail, N.H.; Khan, A.; Shah, S.A.A.; Anwar, A.; Halim, S.A.; Fatmi, M.Q.; Imran, S.; Rahim, F.; Kha, K.M. Synthesis of novel derivatives of oxindole, their urease inhibition and molecular docking studies. Bioorg. Med. Chem. Let. 2015, 25, 3285-3289. [CrossRef] 